\title{
The tidal tails of Milky Way globular clusters
}

\author{
Andrés E. Piatti ${ }^{1,2}$ and Julio A. Carballo-Bello ${ }^{3}$ \\ ${ }^{1}$ Instituto Interdisciplinario de Ciencias Básicas (ICB), CONICET UNCUYO, Padre J. Contreras 1300, (5500) Mendoza, Argentina \\ e-mail: andres.piatti@unc.edu.ar \\ 2 Consejo Nacional de Investigaciones Científicas y Técnicas (CONICET), Godoy Cruz 2290, C1425FQB Buenos Aires, Argentina \\ 3 Instituto de Astrofísica, Facultad de Física, Pontificia Universidad Católica de Chile, Av. Vicuña Mackenna, 4860, $782-0436$ Macul, \\ Santiago, Chile
}

Received 20 March 2020 / Accepted 23 April 2020

\begin{abstract}
We report on the search for overall kinematical or structural conditions that have allowed some Milky Way globular clusters to develop tidal tails. For this purpose, we built a comprehensive catalog of globular clusters with studies focused on their outermost regions and we classify the globular clusters in three categories: those with observed tidal tails, those with extra-tidal features that are different from tidal tails, and those without any signatures of extended stellar density profiles. When exploring different kinematical and structural parameter spaces, we found that globular clusters behave similarly, irrespective of the presence of tidal tails or any other kind of extra-tidal feature, or the absence thereof. In general, globular clusters whose orbits are relatively more eccentric and very inclined, with respect to the Milky Way plane, have undergone a larger amount of mass loss by tidal disruption. The latter has also accelerated the internal dynamics toward a comparatively more advanced stage of evolution. These outcomes show that it is not straightforward to find any particular set of parameter space and dynamical conditions that can definitely predict tidal tails along globular clusters in the Milky Way.
\end{abstract}

Key words. globular clusters: general - methods: observational

\section{Introduction}

The formation of stellar streams or tidal tails due to the disruption or dissolution of Galactic globular clusters has long been understood as a consequence of their interaction with their host galaxy (Grillmair et al. 1995; Dehnen et al. 2004). Indeed, Montuori et al. (2007) performed detailed $N$-body simulations to show that tidal tails are generated in globular clusters as a consequence of their strong interaction with the densest components of the Galaxy, for example, the bulge and the disk, which may result in the presence of multiple tidal tails after the repetitive apocenter passages (Hozumi \& Burkert 2015). However, Küpper et al. (2010, 2012) analytically and numerically show that tidal tails and their substructures originate from the epicyclic motions of a continuous stream of stars escaping the clusters, rather than from tidal shocks, regardless of whether the clusters' orbits are circular or eccentric.

From an observational point of view, there has been a number of studies on the outermost regions of globular clusters with the purpose of finding tidal tails (see, e.g., Malhan et al. 2018; Mateu et al. 2018; Palau \& Miralda-Escudé 2019). The outcomes have been dissimilar since some clusters have been found to have tidal tails, while others have azimuthally (position angle with respect to the globular cluster centers) irregular extended halos or clumpy structures (see, e.g., Moore 1996; Ibata et al. 2013; Kuzma et al. 2016), or simply King (1962) radial profiles without extra-tidal features (see Table 1). Precisely, in this work we carry out a comprehensive compilation of these relevant observational results that have been obtained until now in order to investigate whether there are any cluster structural or internal dynamical properties, and/or orbital parameters that allow us to identify globular clusters with tidal tails.
This work is organized as follows. In Sect. 2 we build a catalog of Milky Way globular clusters with reliable studies on their outermost structures. From this catalog, we analyze, in Sect. 3, different parameter spaces, including kinematical, positional, structural, and internal dynamics properties in order to disentangle any general conditions related to the presence of tidal tails in Milky Way globular clusters. Finally, in Sect. 4 we summarize the main conclusions of this work.

\section{The catalog of extra-tidal features}

We searched the literature looking for studies focused on the structure of the outermost regions of Milky Way globular clusters. In this work, we classify the clusters as G1, G2, and G3, that is, globular clusters with tidal tails, clusters with extratidal features (those that are not symmetric tails, but distributed around the globular clusters), and those without any signature of extended structures, respectively. In G1, we included clusters with clear tails that are extended beyond the cluster's Jacobi radius. Since the Jacobi radius changes between the perigalactic and apogalactic positions, we used the values computed by Piatti et al. (2019) for the semimajor axis of the cluster's orbit (their Eqs. (1) and (3)). Clusters with extra-tidal structures (e.g., azimuthally irregular clumpy features with respect to the globular cluster center, extended halos) are included in the G2 group, while those clusters with stellar radial profiles that do not show any excess beyond the tidal radius given by the model from King (1962) are gathered into the G3 group. We would like to note that making the distinction between $\mathrm{G} 1$ and $\mathrm{G} 2$ groups relies on the shape of their extra-tidal features: while extra-tidal stars are distributed along symmetric tails for G1 clusters, those of G2 clusters are spread around the clusters in halos that are more 
Table 1. Relevant references from the literature of clusters in G1, G2, and G3 groups.

\begin{tabular}{|c|c|c|c|c|c|c|c|c|c|c|c|}
\hline ID & G1 Ref. & G2 Ref. & G3 Ref. & ID & G1 Ref. & G2 Ref. & G3 Ref. & ID & G1 Ref. & G2 Ref. & G3 Ref. \\
\hline NGC 104 & & 6 & & NGC 5694 & & 17 & & NGC 7089 & & & 19 \\
\hline NGC 288 & 1,10 & & & NGC 5824 & & & 30 & NGC 7492 & 22 & & \\
\hline NGC 362 & 25 & & & NGC 5904 & 29 & & & AM 4 & & & 14 \\
\hline NGC 1261 & & 30 & & NGC 6205 & & & 19 & Crater & & & 11 \\
\hline NGC 1851 & 10,20 & & & NGC 6229 & & & 14 & Eridanus & 23 & & \\
\hline NGC 1904 & & 20 & & NGC 6266 & & 13 & & ESO 452-SC11 & & & 7 \\
\hline NGC 2298 & & 18,20 & & NGC 6273 & & 13 & & FSR 1758 & & & 2 \\
\hline NGC 2419 & & 19 & & NGC 6341 & & & 19 & Liller 1 & & & 12 \\
\hline NGC 2808 & & 20 & & NGC 6362 & & 27 & & Pal 1 & 24 & & \\
\hline NGC 3201 & & 15 & & NGC 6544 & & 16 & & Pal 3 & & & 19 \\
\hline NGC 4147 & 19 & & & NGC 6626 & & 13 & & $\mathrm{Pal} 4$ & & & 19 \\
\hline NGC 4590 & 26 & & & NGC 6642 & & 13 & & Pal 5 & 5,28 & & \\
\hline NGC 5024 & & & 14,19 & NGC 6656 & & 15 & & Pal 12 & & & 3 \\
\hline NGC 5053 & & 19 & & NGC 6681 & & 8 & & Pal 14 & 32 & & \\
\hline NGC 5139 & 4,21 & & & NGC 6779 & & 9 & & Pal 15 & 23 & & \\
\hline NGC 5272 & & & 14,19 & NGC 6864 & & & 14 & Rup 106 & & & 14 \\
\hline NGC 5466 & 19,31 & & & NGC 7006 & & 19 & & Whiting 1 & & 14 & \\
\hline NGC 5634 & & 14 & & NGC 7078 & & 19 & & & & & \\
\hline
\end{tabular}

References. (1) Kaderali et al. (2019); (2) Barbá et al. (2019); (3) Musella et al. (2018); (4) Simpson (2020); (5) Odenkirchen et al. (2001); (6) Piatti (2017); (7) Koch et al. (2017); (8) Han et al. (2017); (9) Piatti \& Carballo-Bello (2019); (10) Shipp et al. (2018); (11) Weisz et al. (2016); (12) Saracino et al. (2015); (13) Chun et al. (2015); (14) Carballo-Bello et al. (2014); (15) Kunder et al. (2014); (16) Cohen et al. (2014); (17) Correnti et al. (2011); (18) Balbinot et al. (2011); (19) Jordi \& Grebel (2010); (20) Carballo-Bello et al. (2018); (21) Ibata et al. (2019); (22) Navarrete et al. (2017); (23) Myeong et al. (2017); (24) Niederste-Ostholt et al. (2010); (25) Carballo-Bello (2019); (26) Palau \& MiraldaEscudé (2019); (27) Kundu et al. (2019); (28) Starkman et al. (2020); (29) Grillmair (2019); (30) Kuzma et al. (2018); (31) Belokurov et al. (2006); (32) Sollima et al. (2011).

or less uniformly populated. Such a difference is readily visible while inspecting the stellar density maps and density profiles as a function of the position angle measured from the cluster centers (see references in Table 1).

We found 53 globular clusters with reliable structural information, which represent $\approx 1 / 3$ of those included in the catalog from Harris (1996, 2010 Edition). Although the cluster sample of Table 1 is not complete, the results are useful when trying to find any intrinsic difference between the properties of clusters in the three defined categories. These, in turn, can shed light on our knowledge about the different modes of cluster dissolution. Table 1 does not list every published paper in this field; pioneer works that have been surpassed by recent analysis were omitted.

Table 1 contains 14, 22, and 17 clusters in groups G1, G2, and G3, respectively. Table 1 would seem to suggest that it is really hard to detect tails of debris around globular clusters. Nevertheless, we do not know if every globular cluster should have tidal tails. Globular clusters orbiting very far from the Galactic center do not experience huge tidal forces of Galactic potential, and they then probably never develop stellar debris. Likewise, this observational evidence poses the question as to what conditions may favor a globular cluster to have extra-tidal features. For instance, we can ask whether different kinematical histories (orbits) make a difference in this respect, or whether cluster properties (e.g., size and mass) within certain values are correlated with the existence of tidal tails, among others.

\section{Analysis and discussion}

We started exploring whether the orbital history of globular clusters is related to the occurrence of tidal tails. In this respect, we followed the analysis of Piatti (2019), who found that within the most frequently used orbital properties, the space defined by the eccentricity, the inclination of the orbit, and the semimajor axis (a) turn out to be the best means of shedding light on the overall orbital state of the globular cluster system. For the purposes of our analysis, we added the ratio of the cluster mass that was lost by disruption to the total initial cluster mass $\left(M_{\text {dis }} / M_{\text {ini }}\right)$, as computed by Piatti et al. (2019), to study at what extent the Milky Way gravitational field has shaped the structural parameters and internal dynamics of its globular cluster population. Piatti et al. (2019) estimated how much clusters have been disrupted due to relaxation and tidal heating, and they split the difference between the initial mass $M_{\mathrm{ini}}$ and the current mass $M_{\mathrm{GC}}$, which were both taken from Baumgardt et al. (2019), between mass lost via stellar evolution $\left(M_{\mathrm{ev}}\right)$ and mass lost due to disruption $\left(M_{\mathrm{dis}}\right)$ :

$M_{\mathrm{ini}}=M_{\mathrm{GC}}+M_{\mathrm{ev}}+M_{\mathrm{dis}}$,

with $M_{\mathrm{ev}}=0.5 \times M_{\mathrm{ini}}$, from which they got:

$M_{\mathrm{dis}} / M_{\mathrm{ini}}=1 / 2-M_{\mathrm{GC}} / M_{\mathrm{ini}}$.

We note that $M_{\text {ini }}$ values in Baumgardt et al. (2019) were obtained by integrating each cluster's orbit backwards in time from their observed positions and space velocities and measured current masses, taking into consideration the dynamical drag force. It was additionally assumed that clusters lose half of their $M_{\text {ini }}$ due to stellar evolution during their first gigayear. They iterated over a wide range of $M_{\text {ini }}$ values until they were able to recover each cluster's $M_{\mathrm{GC}}$ on the basis of a linear massloss dependence with time in a spherically symmetric, isothermal galaxy potential over the entire age of each cluster.

Figure 1 shows the relationship between the aforementioned globular cluster parameters. We differentiated clusters in groups G1, G2, and G3 by representing them with filled circles, triangles, and stars, respectively. The ratio of the cluster mass that 


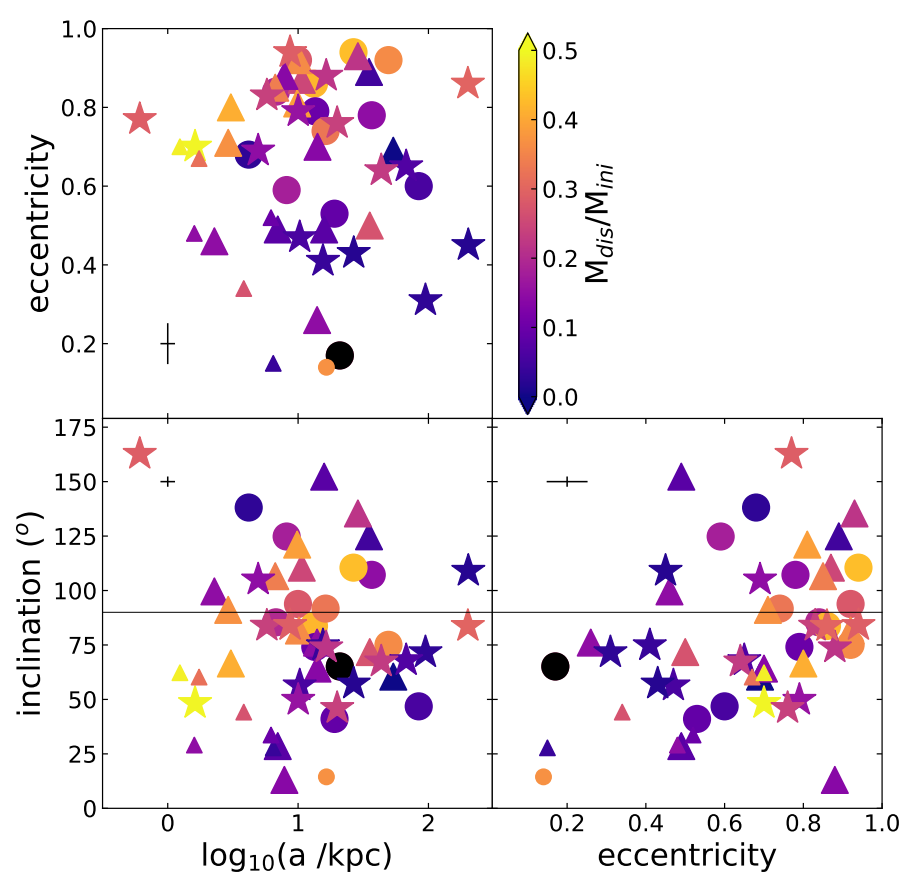

Fig. 1. Relationship between the semimajor axis, the eccentricity, and the orbital inclination for globular clusters in groups G1, G2, and G3, drawn with filled circles, triangles, and stars, respectively. Large and small symbols refer to clusters with an accreted origin or those that formed in situ, respectively, according to Massari et al. (2019). The horizontal line in the bottom panels (inclination $=90^{\circ}$ ) splits them into the prograde (inclination $<90^{\circ}$ ) and retrograde (inclination $>90^{\circ}$ ) regimes (see Piatti 2019). Color bar represents the ratio of the cluster mass lost by disruption to the total initial mass (see Piatti et al. 2019). Error bars are also included. Pal 5 is represented by a large black filled circle.

was lost by disruption to the total cluster mass was used to color the filled symbols as indicated by the adjacent bar. The convention for the orbital inclination is as follows: clusters rotating in prograde orbits, that is, in the direction of the Milky Way rotation, have orbital inclinations $<90^{\circ}$; those in retrograde orbits have inclinations $>90^{\circ}$. We additionally considered the origin of the globular clusters according to Massari et al. (2019): clusters associated with an accreted dwarf galaxy (larger symbols) or those that formed in situ (smaller symbols). We found 1, 6, and 0 clusters formed in situ in groups G1, G2, and G3, respectively.

It can easily be derived from Fig. 1 that, in general terms, there are no clues for distinguishing globular clusters that have tidal tails. Indeed, it would seem that all three defined groups contain clusters that are spread over approximately similar ranges of eccentricity, inclination, and semimajor axis. From this point of view, tidal tails would not seem to arise from the overall kinematical pattern of the globular clusters. However, as can be seen, any globular cluster moving in an orbit with a relatively large eccentricity, that is, along a more radial orbit, has lost a higher amount of its initial mass due to tidal disruption, as compared to globular clusters with smaller eccentricities. Such a behavior is observed in bulge $(\log (a / \mathrm{kpc}) \leq 0.5)$, disk $(0.5<$ $\log (a / \mathrm{kpc}) \leq 1.3))$, and outer halo $(\log (a / \mathrm{kpc})>1.3)$ globular clusters. Nevertheless, a high eccentricity alone would not seem to be enough to produce a large amount of mass loss (see top left panel of Fig. 1).

When a relatively high eccentricity $(\gtrsim 0.8)$ is combined with a very inclined orbit (|inclination $-90^{\circ} \mid \lesssim 20^{\circ}$ ), it is possible to isolate a group of clusters - irrespective of group type (i.e., G1,

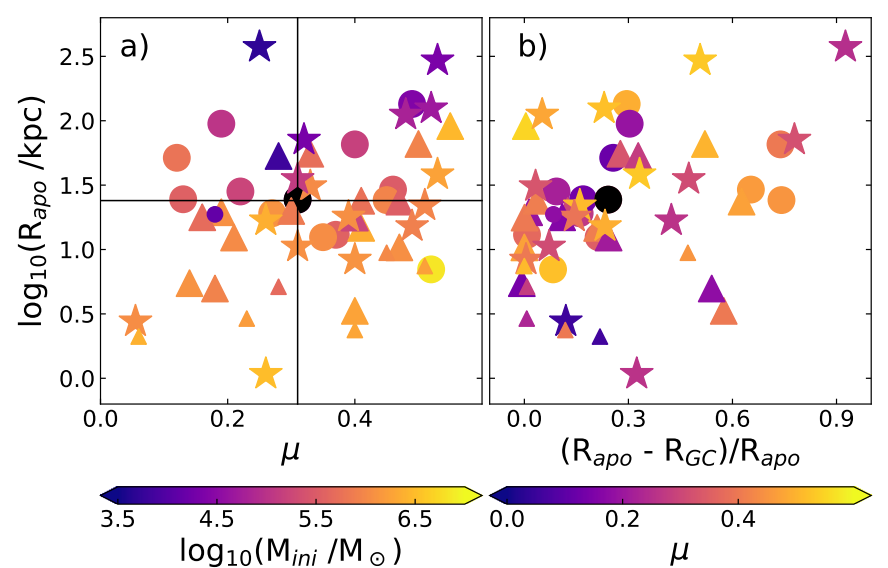

Fig. 2. Relationships between different position and mass-related parameters for the globular clusters in groups G1, G2, and G3. Symbols are the same as in Fig. 1. Panel $a$ is divided into four quadrants as in Fig. 5 from Balbinot \& Gieles (2018). Pal 5 is represented by a large black filled circle.

G2, and G3) - with a relatively large amount of mass lost by disruption (see bottom right panel of Fig. 1). Seemingly, relatively large eccentricities and low orbital inclinations or relatively very inclined orbits and low eccentricities are less efficient in terms of cluster mass tidal disruption. Webb et al. (2014, and reference therein) show that repeated disk passages can contribute to the cluster mass loss by disruption. Hence, a possible scenario that would increase the chances of repeated disk crossing is that of clusters with relatively high eccentricity and inclination values (see Piatti 2019), which could explain the loci of clusters with a disrupted mass that is larger than $\sim 0.3$ in the bottom right panel of Fig. 1.

Balbinot \& Gieles (2018) explore the formation of tidal tails around Milky Way globular clusters from the combination of the fast cluster evolution code Evolve Me A Cluster of StarS (EMACS, Alexander \& Gieles 2012), a semianalytical model for the evolution of the stellar mass function, and a fast stream simulation code. They found that globular clusters with tidal tails are close to dissolution and also likely close to their apogalacticon. We reproduced their Fig. 5 (see Fig. 2a) using the apogalactic distances $\left(R_{\text {apo }}\right)$ from Baumgardt et al. (2019) and the remaining mass fraction ( $\left.\mu=1-M_{\text {dis }} / M_{\text {ini }}\right)$ from Eqs. (4) and (5) of Piatti et al. (2019), which rely on the actual and initial cluster masses computed by Baumgardt et al. (2019). For the sake of the reader, we used the same symbols as in Fig. 1 and subdivided the figure in four panels as in Balbinot \& Gieles (2018).

According to Balbinot \& Gieles (2018), the upper left panel of Fig. 2a encompasses $R_{\text {apo }}$ and $\mu$ values that are more extreme that those for Pal 5, so that globular clusters placed there should be good candidates to develop tidal tails. As can be seen, some globular clusters with observed tidal tails are distributed in that panel, alongside clusters that exhibit extra-tidal structures (G2 group clusters) and also $\mathrm{AM} 4$ at $\approx(0.25,2.6)$, which belong to the G3 group. Therefore, extreme $R_{\text {apo }}$ and $\mu$ values would not be exclusive of G1 group globular clusters. On the other hand, globular clusters with tidal tails also occupy the right-hand panels, that is, those with comparatively smaller amounts of mass loss by tidal disruption. This result suggests that tidal tails can develop early in the cluster disruption process. As for the closeness of their present galactocentric positions $\left(R_{\mathrm{GC}}\right)$ to the respective apogalacticon, Fig. $2 b$ would seem to show that such a condition is not verified by the observations. Indeed, G1 group 


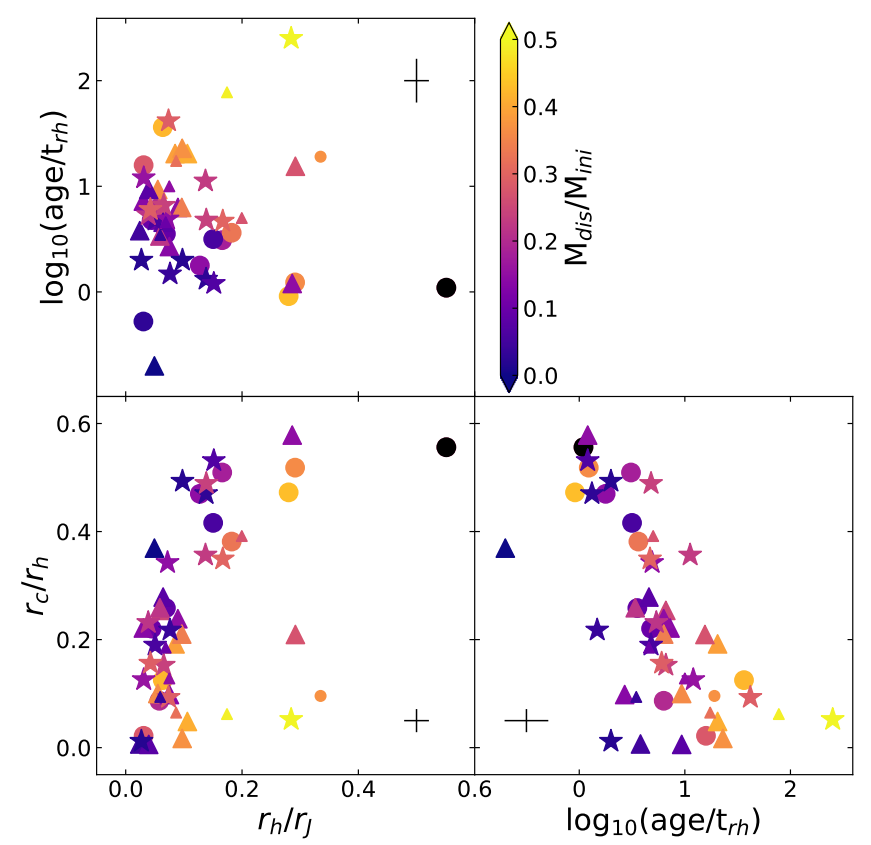

Fig. 3. Relationships between structural and internal dynamical properties of globular clusters in groups G1, G2, and G3. Symbols are the same as in Fig. 1. Pal 5 is represented by a large black filled circle. Negative $\log \left(\right.$ age $\left./ t_{\mathrm{h}}\right)$ comes from adopting an average age for all globular clusters.

clusters can have a remaining mass fraction that is larger than 0.4 and which is located reasonably far from their apogalacticon $\left(\left(R_{\text {apo }}-R_{\mathrm{GC}}\right) / R_{\text {apo }}>0.6\right)$. Additionally, we note that globular clusters with tidal tails are not necessarily those that are more massive initially (see Fig. 2a); although it is known from theory and numerical simulations that the mass-loss rate is a function of the form $\dot{M} \propto-M^{1 / 4} / R_{\mathrm{GC}}$ for globular clusters evolving in an isothermal halo (Gieles et al. 2011). As is also shown in Fig. 1, cluster orbital parameters would not seem to be sufficient in hypothesizing on the presence of tidal tails.

We finally explored whether the presence of tidal tails have any impact in shaping structural and internal dynamical properties, such as core radius $\left(r_{\mathrm{c}}\right)$, half-mass radius $\left(r_{\mathrm{h}}\right)$ and the ratio of the age to the half-mass relaxation time (time required for stars in a system to completely lose the memory of their initial velocity, $t_{\mathrm{h}}$, Spitzer \& Hart 1971). This is motivated by the fact that the mass loss from which the tails are formed could imply a change in the cluster stellar density profile and hence an advanced stage of its internal dynamical evolution (Piatti \& Mackey 2018; Piatti et al. 2019). We used the $r_{\mathrm{c}}, r_{\mathrm{h}}$, and $t_{\mathrm{h}}$ values from Baumgardt et al. (2019), where $t_{\mathrm{h}}$ was calculated using the formalism of Baumgardt \& Hilker (2018). Globular cluster ages were assumed to be $12_{-2.0}^{-1.5} \mathrm{Gyr}$ (Kruijssen et al. 2018). Figure 3 depicts several relationships in which we represent globular clusters in groups G1, G2, and G3 with the same symbols as in Fig. 1. At first glance, globular clusters with observed tidal tails would not seem to differentiate from those with King (1962) profiles. There is a general trend that is common to all three for all three groups of clusters in Table 1 in the sense that: (1) clusters that have lost relatively more mass by disruption do not seem to have preferentially inflated main bodies, although some highly disrupted clusters can be seen at $r_{\mathrm{h}} / r_{J} \gtrsim 0.2$ (see lefthand panels); (2) those that have relatively more compact cores $\left(r_{\mathrm{c}} / r_{\mathrm{h}} \lesssim 0.2\right)$ would seem to be in a more advanced stage of internal dynamical evolution $\left(\log \left(\mathrm{age} / t_{\mathrm{h}}\right) \gtrsim 1.0\right.$, where age $/ t_{\mathrm{h}}$ is a measure of how many times the relaxation time a cluster has lived) (see bottom right panel); and (3) those globular clusters that have relatively expanded cores $\left(r_{\mathrm{c}} / r_{\mathrm{h}} \gtrsim 0.4\right)$ or relatively small main bodies $\left(r_{\mathrm{h}} / r_{J} \lesssim 0.2\right)$ would seem to be in a relatively less advanced stage of dynamical evolution $\left(\log \left(\operatorname{age} / t_{\mathrm{h}}\right) \lesssim 0.7\right)$ (see bottom panels).

\section{Summary and concluding remarks}

The presence of tidal tails in Milky Way globular clusters are witnesses of the interaction experienced by them with their environment while traveling across the Galaxy. Since tidal tails are only observed in some globular clusters, we embarked on an observational-based analysis with the aim of tackling the conditions for whether or not a globular cluster can develop tidal tails.

For this purpose, we gathered reliable information from the literature about the existence of tidal tails, other kinds of extra-tidal structures, such as extended low density halos and azimuthally irregular clumpy features, and the satisfactory fitting of King (1962) models to the outermost regions of the cluster stellar density profiles. From this search we conclude, first of all, that not every globular cluster in the Milky Way has tidal tails. Indeed, from the 53 globular clusters included in our final compilation, 14 have observed tidal tails, 22 have extra-tidal features that are different from tidal tails, and 17 present undetectable signatures of extra-tidal structures.

When exploring kinematical properties (e.g., the orbit's eccentricity, inclination, and semimajor axis) in combination with the ratio of mass lost by disruption to the initial cluster mass, we found that there are no obvious clues to differentiate globular clusters with and without tidal tails. All three defined groups of clusters (G1 for tails, G2 for extra-tidal features, and G3 for King 1962 profiles) have similar kinematical properties. In general, globular clusters that are moving in orbits with a relatively high eccentricity $(\gtrsim 0.8)$ and with very inclined orbits $\left(\approx \pm 70^{\circ}\right.$ from the Milky Way plane) have lost relatively more mass due to tidal disruption than those in more circular and less inclined orbits.

We also found that globular clusters with larger apogalactic distances and a smaller remaining fraction of cluster mass than Pal 5, which is a very well known globular cluster with a long tidal tail highlighted by Balbinot \& Gieles (2018), are not necessarily candidates for developing tidal tails. Furthermore, globular clusters with observed tidal tails are found to keep a larger fraction of the remaining cluster mass and have smaller apogalactic distances than Pal 5. Additionally, globular clusters with extratidal features or King (1962) profiles also span similar ranges of values in the $R_{\text {apo }}$ versus $\mu$ plane. We checked that the initial mass is not correlated with the presence of tidal tails.

Finally, we investigated whether the internal dynamical evolution of globular clusters are reached by the effect of escaping stars in the form of tidal tails. In this respect, we considered different relationships between the core, half-mass and Jacobi radii, the ratio of the cluster age to the respective relaxation time, and the ratio of the mass that was lost due to disruption to the total cluster mass. The outcomes show that irrespective of the presence or the absence of any kind of extra-tidal characteristic, the globular clusters can reach an advanced stage of their internal dynamical evolution if they have lost a relatively large amount of mass by tidal disruption. Therefore, it would seem that there is not any overall property that allows us to predict the presence of tidal tails emerging from a given globular cluster in the Milky Way. 
Acknowledgements. We thank the referee for the thorough reading of the manuscript and timely suggestions to improve it. A.E.P. acknowledge support from the Ministerio de Ciencia, Tecnología e Innovación Productiva (MINCyT) through grant PICT-201-0030. JAC-B acknowledges financial support to CASCONICYT 17003.

\section{References}

Alexander, P. E. R., \& Gieles, M. 2012, EMACSS: Evolve Me A Cluster of StarS Balbinot, E., \& Gieles, M. 2018, MNRAS, 474, 2479

Balbinot, E., Santiago, B. X., da Costa, L. N., Makler, M., \& Maia, M. A. G. 2011, MNRAS, 416, 393

Barbá, R. H., Minniti, D., Geisler, D., et al. 2019, ApJ, 870, L24

Baumgardt, H., \& Hilker, M. 2018, MNRAS, 478, 1520

Baumgardt, H., Hilker, M., Sollima, A., \& Bellini, A. 2019, MNRAS, 482, 5138

Belokurov, V., Evans, N. W., Irwin, M. J., Hewett, P. C., \& Wilkinson, M. I 2006, ApJ, 637, L29

Carballo-Bello, J. A. 2019, MNRAS, 486, 1667

Carballo-Bello, J. A., Sollima, A., Martínez-Delgado, D., et al. 2014, MNRAS 445,2971

Carballo-Bello, J. A., Martínez-Delgado, D., Navarrete, C., et al. 2018, MNRAS, 474,683

Chun, S.-H., Kang, M., Jung, D., \& Sohn, Y.-J. 2015, AJ, 149, 29

Cohen, R. E., Mauro, F., Geisler, D., et al. 2014, AJ, 148, 18

Correnti, M., Bellazzini, M., Dalessandro, E., et al. 2011, MNRAS, 417, 2411

Dehnen, W., Odenkirchen, M., Grebel, E. K., \& Rix, H.-W. 2004, AJ, 127, 2753

Gieles, M., Heggie, D. C., \& Zhao, H. 2011, MNRAS, 413, 2509

Grillmair, C. J. 2019, ApJ, 884, 174

Grillmair, C. J., Freeman, K. C., Irwin, M., \& Quinn, P. J. 1995, AJ, 109, 2553

Han, M., Chun, S.-H., Choudhury, S., et al. 2017, J. Astron. Space Sci., 34, 83

Harris, W. E. 1996, AJ, 112, 1487

Hozumi, S., \& Burkert, A. 2015, MNRAS, 446, 3100

Ibata, R., Nipoti, C., Sollima, A., et al. 2013, MNRAS, 428, 3648

Ibata, R. A., Bellazzini, M., Malhan, K., Martin, N., \& Bianchini, P. 2019, Nat. Astron., 3, 667

Jordi, K., \& Grebel, E. K. 2010, A\&A, 522, A71

Kaderali, S., Hunt, J. A. S., Webb, J. J., Price-Jones, N., \& Carlberg, R. 2019, MNRAS, 484, L114
King, I. 1962, AJ, 67, 471

Koch, A., Hansen, C. J., \& Kunder, A. 2017, A\&A, 604, A41

Kruijssen, J. M. D., Pfeffer, J. L., Reina-Campos, M., Crain, R. A., \& Bastian, N. 2018, MNRAS

Kunder, A., Bono, G., Piffl, T., et al. 2014, A\&A, 572, A30

Kundu, R., Minniti, D., \& Singh, H. P. 2019, MNRAS, 483, 1737

Küpper, A. H. W., Kroupa, P., Baumgardt, H., \& Heggie, D. C. 2010, MNRAS, 401, 105

Küpper, A. H. W., Lane, R. R., \& Heggie, D. C. 2012, MNRAS, 420, 2700

Kuzma, P. B., Da Costa, G. S., Mackey, A. D., \& Roderick, T. A. 2016, MNRAS, 461, 3639

Kuzma, P. B., Da Costa, G. S., \& Mackey, A. D. 2018, MNRAS, 473, 2881

Malhan, K., Ibata, R. A., \& Martin, N. F. 2018, MNRAS, 481, 3442

Massari, D., Koppelman, H. H., \& Helmi, A. 2019, A\&A, 630, L4

Mateu, C., Read, J. I., \& Kawata, D. 2018, MNRAS, 474, 4112

Montuori, M., Capuzzo-Dolcetta, R., Di Matteo, P., Lepinette, A., \& Miocchi, P. 2007, ApJ, 659, 1212

Moore, B. 1996, ApJ, 461, L13

Musella, I., Di Criscienzo, M., Marconi, M., et al. 2018, MNRAS, 473, 3062

Myeong, G. C., Jerjen, H., Mackey, D., \& Da Costa, G. S. 2017, ApJ, 840, L25

Navarrete, C., Belokurov, V., \& Koposov, S. E. 2017, ApJ, 841, L23

Niederste-Ostholt, M., Belokurov, V., Evans, N. W., et al. 2010, MNRAS, 408 , L66

Odenkirchen, M., Grebel, E. K., Rockosi, C. M., et al. 2001, ApJ, 548, L165

Palau, C. G., \& Miralda-Escudé, J. 2019, MNRAS, 488, 1535

Piatti, A. E. 2017, ApJ, 846, L10

Piatti, A. E. 2019, ApJ, 882, 98

Piatti, A. E., \& Carballo-Bello, J. A. 2019, MNRAS, 485, 1029

Piatti, A. E., \& Mackey, A. D. 2018, MNRAS, 478, 2164

Piatti, A. E., Webb, J. J., \& Carlberg, R. G. 2019, MNRAS, 489, 4367

Saracino, S., Dalessandro, E., Ferraro, F. R., et al. 2015, ApJ, 806, 152

Shipp, N., Drlica-Wagner, A., Balbinot, E., et al. 2018, ApJ, 862, 114

Simpson, J. D. 2020, MNRAS, 491, 3374

Sollima, A., Martínez-Delgado, D., Valls-Gabaud, D., \& Peñarrubia, J. 2011, ApJ, 726, 47

Spitzer, L., Jr, \& Hart, M. H. 1971, ApJ, 164, 399

Starkman, N., Bovy, J., \& Webb, J. 2020, MNRAS, 493, 4978

Webb, J. J., Sills, A., Harris, W. E., \& Hurley, J. R. 2014, MNRAS, 445, 1048

Weisz, D. R., Koposov, S. E., Dolphin, A. E., et al. 2016, ApJ, 822, 32 